\title{
Erratum to: Effects of fish farming on plankton structure in a Brazilian tropical reservoir
}

\author{
Paula A. F. Borges - Sueli Train • \\ Juliana D. Dias · Cláudia C. Bonecker
}

Published online: 21 May 2010

(C) Springer Science+Business Media B.V. 2010

\section{Erratum to: Hydrobiologia \\ DOI 10.1007/s10750-010-0271-2}

On page 10, column 1, lines 9-10, please replace "according to the Ordinance from the the Brazilian Health Ministry 518/2004 (Brasil, 2004)." by "according to recommendations from the Brazilian Health Ministry (Brasil, 2003)."

Please delete the reference:

"Brasil. 2004. Ministério da Saúde. Portaria no. 518. Estabelece os procedimentos e responsabilidades relativos ao controle e vigilância da qualidade da água para consumo humano e seus padrões de potabilidade, e dá outras providências."

and replace it by:

"Brasil. 2003. Cianobactérias tóxicas na água para consumo humano na saúde pública e processos de remoção em água para consumo humano. Ministério da Saúde. Fundação Nacional de Saúde, Brasília, DF."

The online version of the original article can be found under doi:10.1007/s10750-010-0271-2.

P. A. F. Borges $(\bowtie) \cdot$ S. Train · J. D. Dias .

C. C. Bonecker

Programa de Pós-Graduação em Ecologia de Ambientes Aquáticos Continentais (PEA), Núcleo de Pesquisas em Limnologia, Ictiologia e Aqüicultura (Nupélia),

Departamento de Biologia, Universidade Estadual de Maringá, Av. Colombo, 5790, 87020-900 Maringá, PR, Brazil

e-mail: pafborges@gmail.com

P. A. F. Borges

Laboratório de Biologia Molecular, Departamento de Zoologia, Universidade Federal do Paraná, Curitiba, PR, Brazil 